\title{
Evolutionary Cycling in Predator-Prey Interactions: Population Dynamics and the Red Queen
}

\author{
Ulf Dieckmann $\dagger+$ Paul Marrow $\$ \|$ and Richard Law \\ $\dagger$ Arbeitsgruppe Theoretische Ökologie, Forschungszentrum Jülich GmbH, Postfach 1913, \\ 52425 Jülich, F.R.G., $\$$ Institute of Ecological and Evolutionary Sciences, Leiden University, \\ Kaiserstraat 63, 2311 GP Leiden, The Netherlands and § Department of Biology, \\ University of York, York YO1 5DD, U.K.
}

(Received on 21 October 1994, Accepted on 26 April 1995)

\begin{abstract}
This paper describes the coevolution of phenotypes in a community comprising a population of predators and of prey. It is shown that evolutionary cycling is a likely outcome of the process. The dynamical systems on which this description is based are constructed from microscopic stochastic birth and death events, together with a process of random mutation. Births and deaths are caused in part by phenotype-dependent interactions between predator and prey individuals and therefore generate natural selection. Three outcomes of evolution are demonstrated. A community may evolve to a state at which the predator becomes extinct, or to one at which the species coexist with constant phenotypic values, or the species may coexist with cyclic changes in phenotypic values. The last outcome corresponds to a Red Queen dynamic, in which the selection pressures arising from the predator-prey interaction cause the species to evolve without ever reaching an equilibrium phenotypic state. The Red Queen dynamic requires an intermediate harvesting efficiency of the prey by the predator and sufficiently high evolutionary rate constant of the prey, and is robust when the model is made stochastic and phenotypically polymorphic. A cyclic outcome lies outside the contemporary focus on evolutionary equilibria, and argues for an extension to a dynamical framework for describing the asymptotic states of evolution.
\end{abstract}

(C) 1995 Academic Press Limited

\section{Introduction}

Predator-prey interactions are ubiquitous in nature (Crawley, 1992). Sometimes the ecological interactions between predator and prey species can be strong enough for the predator to be a major component of the environment in which the prey is evolving, and vice versa. Such interactions have therefore motivated a variety of theoretical models of phenotypic coevolution in predator-prey communities (e.g. Rosenzweig, 1973; Parker, 1985; Abrams, 1986; Brown \& Vincent, 1992). Of some interest has been the question of whether the phenotypes of the predator and prey evolve to an equilibrium asymptotic state, such as an evolutionarily stable strategy (Maynard Smith \& Price,

\| Present address: Large Animal Research Group, Department of Zoology, University of Cambridge, Downing Street, Cambridge CB2 3EJ, U.K.
1973). An alternative could be that their interaction prevents attainment of an equilibrium point and that there is continuous evolutionary change of their phenotypes. Following Van Valen's (1973) Red Queen hypothesis, the latter behaviour has become known as Red Queen dynamics (Stenseth \& Maynard Smith, 1984; Rosenzweig et al., 1987; Marrow et al., 1992). To make this notion precise, we refer here to a Red Queen dynamic as any phenotypic dynamic that, in the absence of external forcing, does not tend to an equilibrium state.

In the literature, it has been argued that a Red Queen dynamic would require the set of feasible phenotypes to be unbounded, so that the phenotypes could evolve to ever more extreme states. Rosenzweig et al. (1987) concluded that "the Red Queen depends on the existence of special phenotypic features, i.e. those which are independent, boundless, and about which it 
may be said, the larger (or smaller, or denser, or furrier, or...), the better". This requirement is unlikely to be met in reality, and calls into question whether Red Queen dynamics could occur at all. To investigate whether Red Queen dynamics are possible, we have developed models of the evolutionary dynamics of predator and prey phenotypes (Marrow, 1992; Marrow et al., 1992; Marrow \& Cannings, 1993). These models suggested that, over the course of evolution, the phenotypes could either tend to equilibrium or to non-equilibrium asymptotic states. The models did not incorporate time explicitly, and for this reason could give only qualitative information on the direction of evolution. To determine the asymptotic states of coevolving systems, it is necessary to build the time-dependent processes into the framework of a dynamical system (Dieckmann \& Law, 1995; Marrow et al., 1995).

In this paper, we utilize a hierarchy of three dynamical models to investigate the phenotypic states to which coevolving predators and prey could tend. These models represent different balances between descriptive capacity and corresponding analytic tractability. Mathematical details are given in the Appendix. Section 2 introduces the ecological interactions which define the predator-prey community, and Section 3 briefly explains the distinctive features of the three models used. In Section 4 we demonstrate that the system eventually attains one of three different evolutionary states: (i) the predator goes extinct, (ii) coevolution leads to constant phenotypes in predator and prey, and (iii) the phenotypes in both species undergo coupled and sustained oscillations on a limit cycle corresponding to Red Queen dynamics. Section 5 analyses the requirements for this evolutionary cycling. The dependence of cycling on the interaction and mutation structure of the predator and prey is revealed, and we show that the phenomenon is robust under changes in the modelling approach. To our knowledge this is the first example of a Red Queen coevolutionary process driven by population dynamic change (corresponding to the second mechanism proposed by Stenseth \& Maynard Smith, 1984). We conclude that the conceptual framework of phenotypic evolution, with its current focus on fixed points (like evolutionarily stable strategies) as the endpoints of evolution, needs to be expanded to encompass more complex evolutionary attractors such as the limit cycles presented here.

\section{The Coevolutionary Community}

Our models of phenotypic evolution are underpinned by ecological processes describing the dynamics of predator and prey populations. This ensures that the process of natural selection directing evolution is driven explicitly by the ecology of predator-prey interactions, rather than by an external ad hoc notion of relative fitness of different phenotypes. For simplicity, we focus on a single phenotypic trait in each species; in view of the importance of body size in determining interactions between predator and prey (Cohen et al., 1993), one might think of these traits as body sizes, $s_{1}$ and $s_{2}$, of prey and predator, respectively.

To describe the population dynamics in our community, it is necessary to define the ecological processes that affect the population sizes of the two species (Table 1). Table 1(a) describes the birth and death events that are dependent on phenotype, these being the events that arise from encounters with other individuals, as opposed to the constant birth and death events given in Table 1(b).

Evolutionary processes in the community require a mechanism for generating phenotypic variation on which natural selection caused by the interaction between predator and prey can operate. We assume that variation is created by a simple mutation process; in order to keep the analysis tractable we envisage that the genetic systems of the species are clonal. Table 1(c) shows that each birth event gives rise with probabilities $\mu_{1}$ and $\mu_{2}$ to a mutant offspring in the phenotypic traits, $s_{1}$ and $s_{2}$, of prey and predator, respectively. The new phenotypes are chosen according to the mutation distributions, $M_{1}$ and $M_{2}$, of prey and predator, respectively.

Natural selection arises from the dependence of the birth and death probabilities per unit time $\alpha, \beta$ and $\gamma$ on the phenotypes of the interacting individuals. Various functions could be used; we use functions as described in Fig. 1. Thus the function $\alpha$, which characterizes the ecological processes responsible for self-limitation in the prey's population size, is taken to be parabolic such that intermediate phenotypes are favoured in the absence of the predator [Fig. 1(a)]. The function $\beta$ describing the effect of a predator on the probability of death of the prey is taken to be bivariate Gaussian [Fig. 1(b)], on the grounds that the predator is likely to show some degree of specialization in the size of prey it chooses relative to its own size (Cohen et al., 1993). On the basis that what is bad for the prey is good for the predator, the function $\gamma$ is related to $\beta$ by a constant of proportionality, $\gamma=h \cdot \beta$. We call $h$ the harvesting efficiency.

The ecological community presented here extends the model of Marrow et al. (1992) by providing a full dynamical description of the birth, death and 
TABLE 1

Definition of birth, death and mutation processes for a prey individual of size $s_{1}$ and predator of size $s_{2}$

(a) Birth and death processes affected by phenotype

\begin{tabular}{lllc}
\hline $\begin{array}{l}\text { Target } \\
\text { individual }\end{array}$ & $\begin{array}{c}\text { Encountered } \\
\text { individual }\end{array}$ & Birth/death event & $\begin{array}{c}\text { Probability of event } \\
\text { per encounter per unit time }\end{array}$ \\
\hline 1. prey $s_{1}$ & prey $\tilde{s}_{1}$ & death of prey $s_{1}$ & $\alpha\left(s_{1}\right) \dagger$ \\
2. prey $s_{1}$ & predator $s_{2}$ & death of prey $s_{1}$ & $\beta\left(s_{1}, s_{2}\right)$ \\
3. predator $s_{2}$ & prey $s_{1}$ & birth of predator $s_{2}$ & $\gamma\left(s_{1}, s_{2}\right)$ \\
\hline
\end{tabular}

(b) Birth and death processes independent of phenotype

\begin{tabular}{lcc}
\hline Target individual & Birth/death event & $\begin{array}{c}\text { Probability of event } \\
\text { per capita per unit time }\end{array}$ \\
\hline $\begin{array}{l}\text { 1. prey } s_{1} \\
\text { 2. predator } s_{2}\end{array}$ & $\begin{array}{l}\text { birth of prey } s_{1} \\
\text { death of predator } s_{2}\end{array}$ & $r_{1}$ \\
\hline (c) Mutation processes & & $r_{2}$ \\
\hline Birth event & Mutation event & Probability distribution $t$ \\
\hline 1. birth of prey $s_{1}$ & prey $s_{1} \rightarrow s_{1}^{\prime}$ & $\left(1-\mu_{1}\right) \delta\left(s_{1}^{\prime}-s_{1}\right)+\mu_{1} M_{1}\left(s_{1}^{\prime}-s_{1}\right)$ \\
2. birth of predator $s_{2}$ & predator $s_{2} \rightarrow s_{2}^{\prime}$ & $\left(1-\mu_{2}\right) \delta\left(s_{2}^{\prime}-s_{2}\right)+\mu_{2} M_{2}\left(s_{2}^{\prime}-s_{2}\right)$ \\
\hline
\end{tabular}

$\dagger$ This death event is taken to be dependent only on the phenotype $s_{1}$ of the target individual, not on that of the encountered individual $\tilde{s}_{1}$.

$\$ \mu_{i}$ is the probability that the birth event in species $i$ is a mutant; $\delta$ is the Dirac $\delta$ function; $M_{i}$ is the mutation distribution.

mutation processes. It generalizes the former account in the sense that (i) it allows stochastic population dynamics arising from individual-based encounters, and (ii) it permits the populations to have polymorphic phenotypic distributions since multiple phenotypic trait values may be present simultaneously in each species. From Table 1 we recover as a special case the well-known Lotka-Volterra equations

$$
\begin{gathered}
\dot{n}_{1}=n_{1}\left(r_{1}-\alpha\left(s_{1}\right) n_{1}-\beta\left(s_{1}, s_{2}\right) n_{2}\right) \\
\dot{n}_{2}=n_{2}\left(-r_{2}+\gamma\left(s_{1}, s_{2}\right) n_{1}\right)
\end{gathered}
$$

(a)

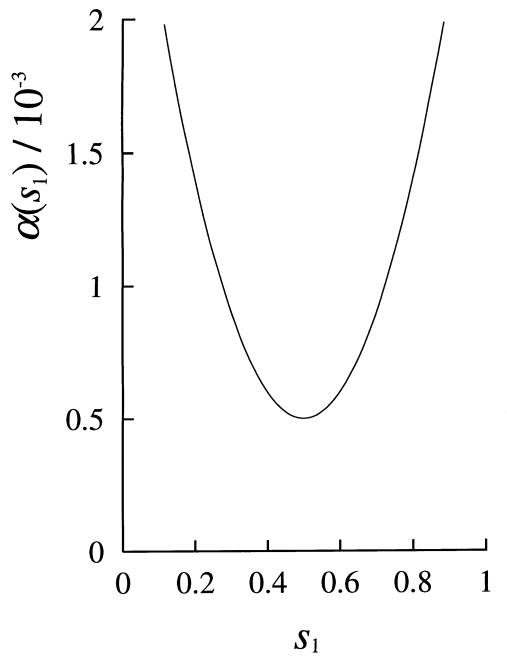

(b)

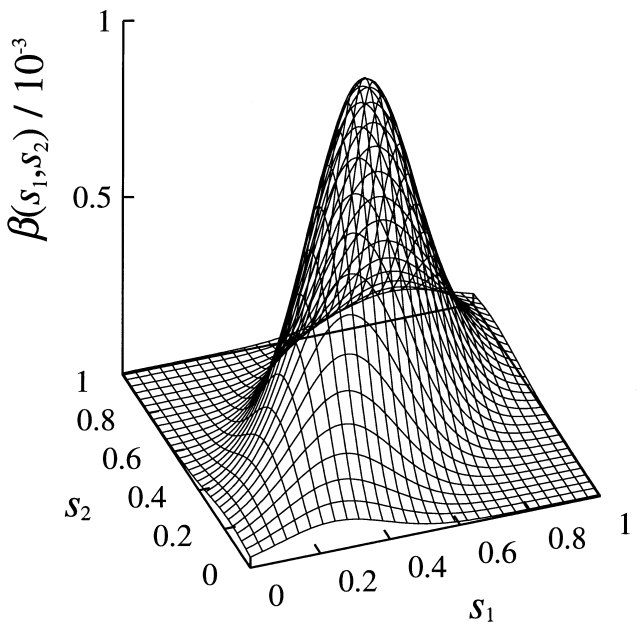

FIG. 1. Specification of the coevolutionary community given in Table 1 . The functions used to describe the effect of phenotype $\left(s_{1}, s_{2}\right)$ on the birth and death probabilities arising from encounters between individuals are: (a) prey self-limitation $\alpha\left(s_{1}\right) / u=c_{1}-c_{2} s_{1}+c_{3} s_{1}^{2}$, (b) effect of predator on prey $\beta\left(s_{1}, s_{2}\right) / u=\exp \left(-\delta_{1}^{2}+2 c_{4} \delta_{1} \delta_{2}-\delta_{2}^{2}\right)$, where $\delta_{1}=\left(s_{1}-c_{5}\right) / c_{6}$ and $\delta_{2}=\left(s_{2}-c_{7}\right) / c_{8}$, and $u$ is a constant that scales population sizes. Parameters take the values: $c_{1}=3.0, c_{2}=10.0, c_{3}=10.0, c_{4}=0.6, c_{5}=0.5, c_{6}=0.22, c_{7}=0.5, c_{8}=0.25$. The function $\gamma\left(s_{1}, s_{2}\right)$ is not shown since it is related to $\beta\left(s_{1}, s_{2}\right)$ by the constant of proportionality $h$. The constant birth and death terms are: $r_{1}=0.5, r_{2}=0.05$. Mutation parameters used in the paper are: $\mu_{1}=10^{-3}, \mu_{2}=10^{-3} ; M_{1}$ and $M_{2}$ are normal distributions with mean 0 and $\sqrt{\operatorname{var} M_{1}}=2 \times 10^{-3}, \sqrt{\operatorname{var} M_{2}}=2 \times 10^{-3}$, except where otherwise stated. The quantity $u=10^{-3}$ is constant throughout. 
for the population sizes, $n_{1}$ and $n_{2}$, of prey and predator, respectively, by assuming no mutations, random encounters, deterministic population dynamics (the population sizes of the species are large), and monomorphic phenotypic distributions (only one phenotype present within each species).

\section{Three Dynamical Models Of Coevolution}

Equations 1 illustrate how the general coevolutionary process defined in Table 1 can be reduced by making appropriate simplifying assumptions. In a similar spirit, three dynamical models are derived in the Appendix for the change in phenotypic traits $s_{1}$ and $s_{2}$ of the prey and predator respectively.

1. Polymorphic stochastic model. This provides a full description of the dynamics defined in Table 1. It can be given as a multivariate functional master equation, and depends only on the assumption of random encounters, thus allowing both for polymorphism and for stochasticity.

2. Monomorphic stochastic model. This retains the stochasticity in the coevolutionary process, but assumes that variation in the phenotypic distributions is small enough for an assumption of monomorphism to provide a good approximation. The coevolutionary process can then be described as a directed random walk in the phenotype space spanned by $s_{1}$ and $s_{2}$. Stochastic steps occur when a resident phenotype is replaced by an advantageous mutant, e.g. $s_{1} \rightarrow s_{1}^{\prime}$; a sequence of such substitutions is called a trait substitution sequence (Metz et al., 1992). The model is framed as a multivariate master equation.

3. Monomorphic deterministic model. This is a deterministic approximation to the monomorphic stochastic model above. It is given in terms of a system of ordinary differential equations describing the expected evolutionary paths in the phenotype space.

Further information as to the relation between the three models is given in the Appendix and in Dreckmann (1994). The full derivation of the monomorphic models is given in Dieckmann \& Law (1995), and a discussion of the third model can be found in Marrow et al. (1995).

\section{Evolutionary Outcomes}

In this section we describe the variety of possible evolutionary outcomes in a predator-prey community, using the monomorphic deterministic model. Deterministic dynamics of this kind have been used elsewhere in the literature (e.g. Hofbauer \& Sigmund, 1990; Vincent, 1991; Abrams et al., 1993), but have not previously been underpinned by a formal derivation.

In the case of the monomorphic phenotypic dynamics, we can immediately infer from eqns (1) that there is a region in the phenotype space where both species can coexist with positive population densities. The boundary of this region is depicted by the oval discontinuous curves in Figure 2. Only within this region can the predator population harvest the prey sufficiently to survive; given a pair of phenotypes $\left(s_{1}, s_{2}\right)$ outside this region, the predator population is driven to extinction by the population dynamics (1). Accordingly, coevolution of the predator and prey can only be observed within this region of coexistence.

For a coevolving predator-prey community starting with phenotypes in the region of coexistence, there are eventually three possible outcomes.

1. Evolution to a fixed point. In Fig. 2(a) the phenotypic values tend to an equilibrium point; once this is reached, no further evolution occurs. There are in fact three fixed points at the intersection of the isoclines (i.e. at $\dot{s}_{1}=0, \dot{s}_{2}=0$, see Appendix) in this example, as can be seen from the accompanying phase portrait [Fig. 2(b)]; two of these are attractors and they are separated by the stable manifold of the third which is a saddle point. Notice that the coevolutionary process here is multistable with two attractors having disjunct domains of attraction; thus there may be no more reason for a particular observed asymptotic evolutionary state than the more or less arbitrary initial conditions.

2. Evolution to extinction. In Fig. 2(c) the coevolutionary process drives the phenotypic values towards the boundary of the region of coexistence [see Fig. 2(d)]. There the predator population goes extinct and the predator phenotype is no longer defined. The phenotype space of the community collapses from $\left(s_{1}, s_{2}\right)$ to the one dimensional space $s_{1}$, where the prey phenotype continues to evolve to its own equilibrium point. Note here that the extinction of the predator is driven by the evolutionary dynamics in $\left(s_{1}, s_{2}\right)$ and not merely by the population dynamics in $\left(n_{1}, n_{2}\right)$.

3. Evolutionary cycling. In Fig. 3(a) the coevolutionary process in the predator-prey community continues indefinitely; mutants replace residents in a cyclic manner such that the phenotypes eventually return to their original values and do not reach an equilibrium point. As can be seen from Fig. 3(b), the attractor is a limit cycle, confirming the conjecture 
(a)

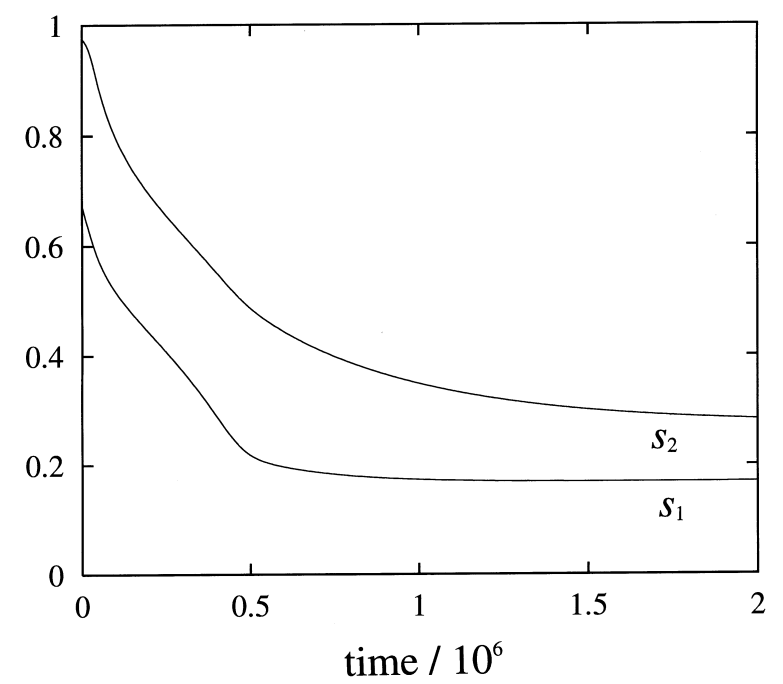

(c)

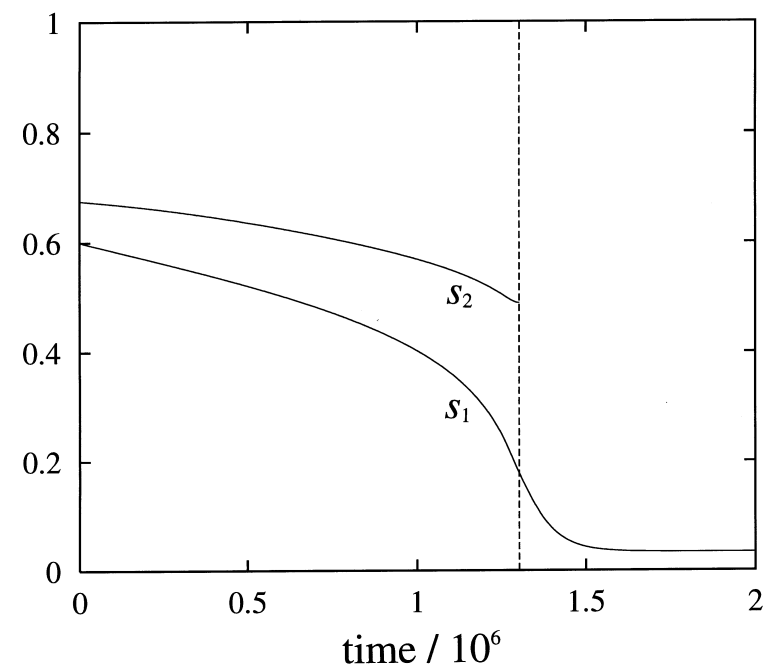

(b)

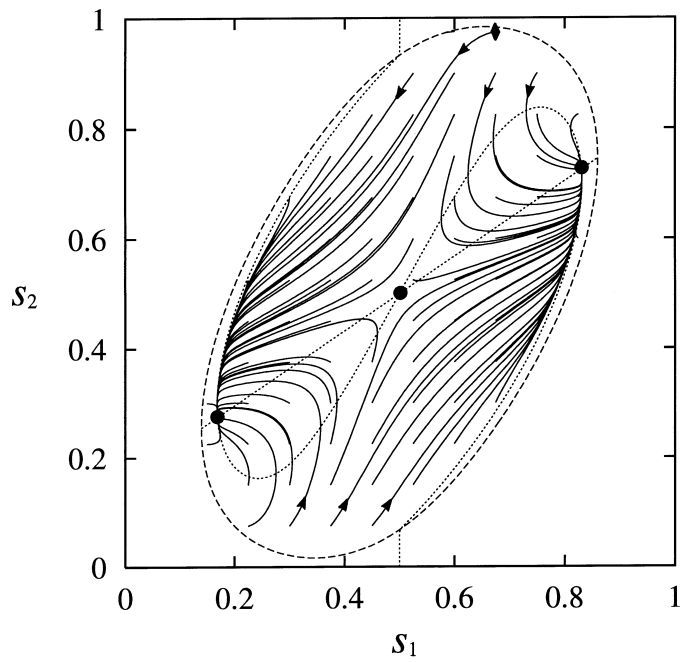

(d)

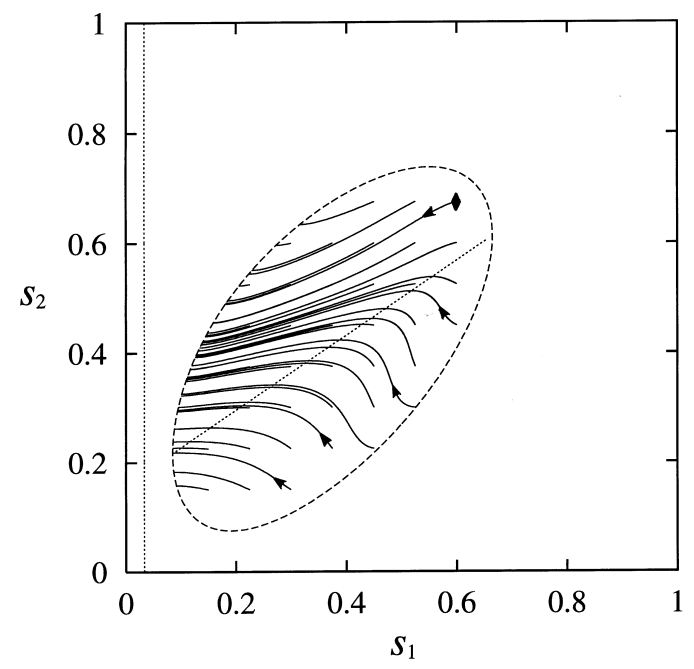

FIG. 2. Patterns of evolution of prey $\left(s_{1}\right)$ and predator $\left(s_{2}\right)$ phenotypes obtained from the monomorphic deterministic model. (a) Solution that tends to an equilibrium point over the course of time obtained using the parameter values in figure 1 with $h=1$. (b) Phase portrait of the phenotype space from which (a) is drawn with orbits shown as continuous lines in the direction indicated by the arrows: the starting point of the orbit corresponding to the solution in (a) is shown as the diamond. The boundary of the region of coexistence of the predator and prey is given as the discontinuous oval line. Isoclines are shown as dotted lines (straight line: predator; curved line: prey); equilibrium points occur at the intersection of the isoclines and are indicated by the filled circles. (c) Solution for a community that evolves to predator extinction at time $=1.30 \times 10^{6}$. After this time, the prey continues to evolve in the absence of the predator. Parameter values as in figure 1 , except $c_{1}=1.0$, $c_{2}=1.0, c_{3}=15.0$, and with $h=1$. (d) Phase portrait of the phenotype space from which solution (c) is drawn; the starting point of the orbit corresponding to the solution in (c) is shown as the diamond. The prey isocline lies outside the region of coexistence and orbits touch the boundary of the region of coexistence at which point the predator goes extinct.

made by Marrow et al. (1992) that Red Queen coevolution can occur in this predator-prey community.

These three outcomes of coevolution correspond to the endpoints of evolutionary arms races discussed qualitatively by Dawkins \& Krebs (1979), namely: (i) equilibrium endpoints, (ii) one side wins, and (iii) cyclic endings.

\section{Requirements For Cycling}

Here, we investigate the robustness of the phenomenon of evolutionary cycling. We do this in two ways. First a bifurcation analysis of the monomorphic deterministic model is given; this allows one to establish the range of parameters in the model that permit evolutionary cycling to occur. Second, we examine the monomorphic stochastic model and 
(a)

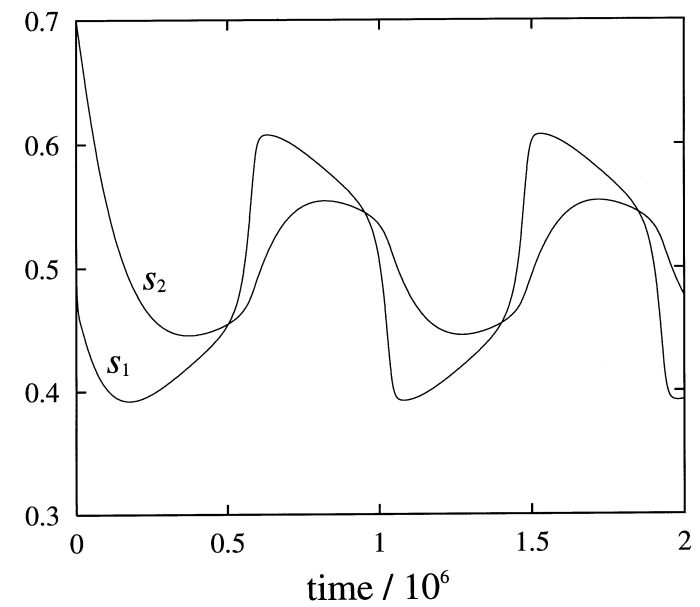

(c)

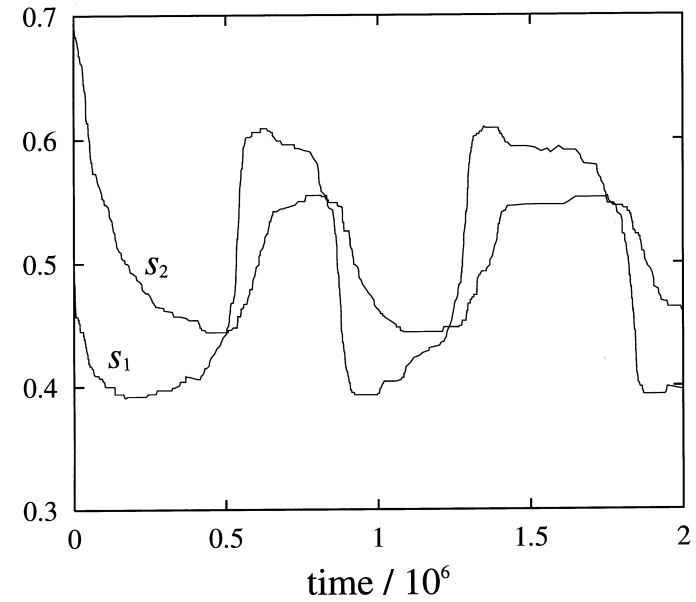

(e)

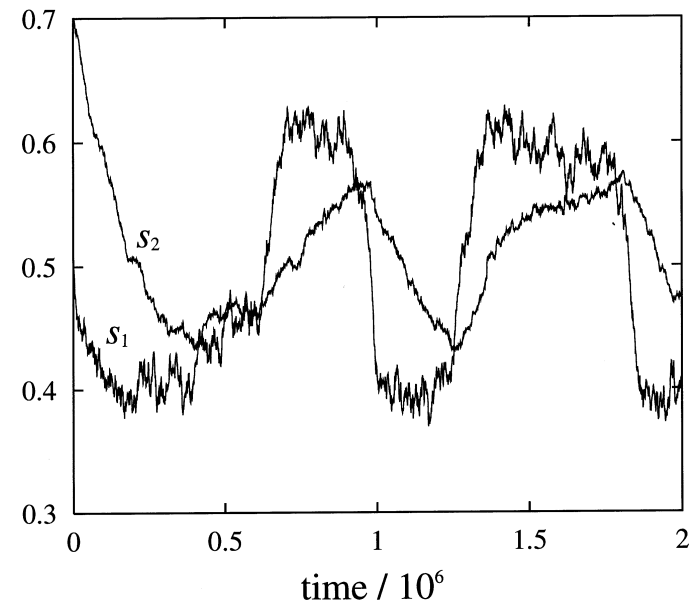

(b)

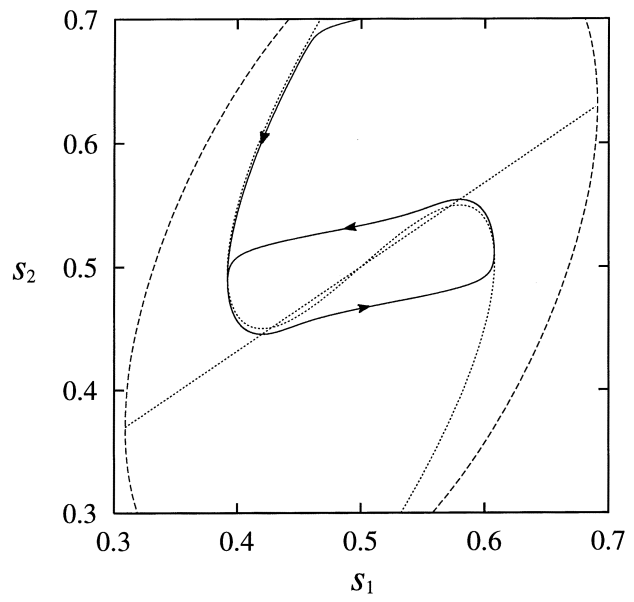

(d)

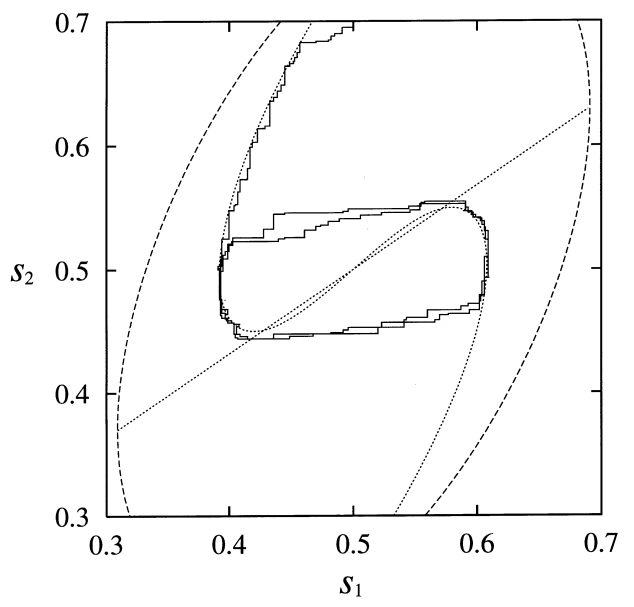

(f)

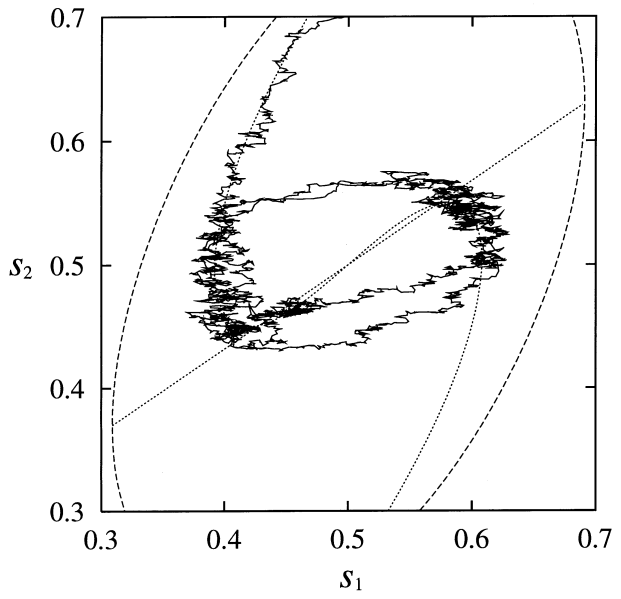

FIG. 3. Example of evolutionary cycling using the monomorphic deterministic model in (a) and (b), the monomorphic stochastic model in (c) and (d), and the polymorphic stochastic model in (e) and (f). Graphs (a), (c) and (e) show the values of the prey $\left(s_{1}\right)$ and predator $\left(s_{2}\right)$ phenotypes as functions of time (mean values in the case of the polymorphic model). The corresponding orbits are shown as continuous lines in the phase spaces given in graphs (b), (d) and (f). See Fig. 2 for an explanation of the phase portrait. Parameter values for these simulations are identical and are set as given in figure 1 , except $\mu_{1}=10^{-2}, \mu_{2}=10^{-2}$ and with $h=0.14$. 


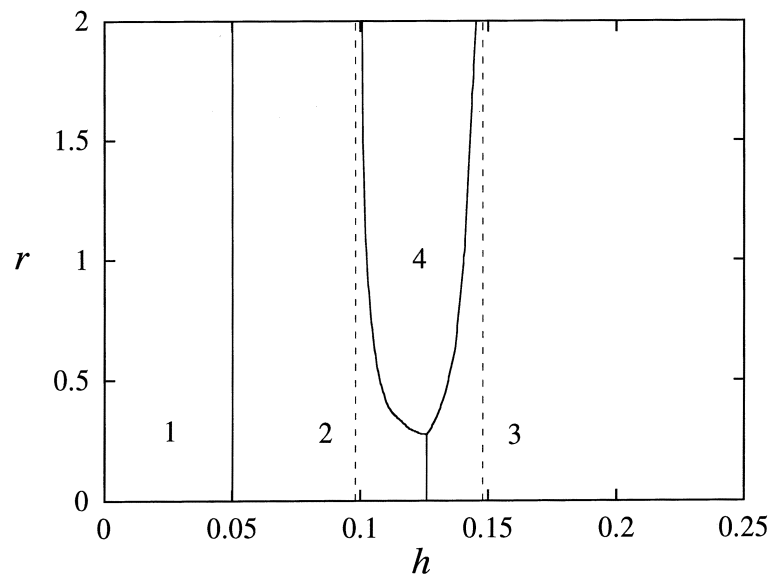

FIG. 4. Results of bifurcation analysis, showing the effect of the harvesting efficiency $h$, and the ratio of the evolutionary rate constants $r$ on the dynamics of the monomorphic deterministic model. Regions are: (1) predator absent, (2) one fixed point present, which is an attractor, (3) three fixed points, two of which are attractors, (4) limit cycle attractor.

finally the polymorphic stochastic model to see how robust the phenomenon of evolutionary cycling is when the simplifying assumptions of the monomorphic deterministic model are removed.

\section{BIFURCATION ANALYSIS OF THE MONOMORPHIC DETERMINISTIC MODEL}

We focus on the effect of two quantities of particular interest from an ecological viewpoint. These are, first, the predator's efficiency in harvesting the prey as given by the ratio $h=\gamma / \beta$, and, second, the ratio of the evolutionary rate constants $r=\left(\mu_{1} \operatorname{var} M_{1}\right) /$ $\left(\mu_{2} \operatorname{var} M_{2}\right)$ (see Appendix). The results of the bifurcation analysis are presented in Fig. 4. Four distinct regions within the parameter space can be seen:

1. For $h<5 \%$ the two species cannot coexist, and therefore no coevolution can occur.

2. For $5 \%<h<9.8 \%$ there exists only one fixed point for the monomorphic deterministic model. This fixed point is an attractor; the system evolves to this point and there is no further coevolution once it is reached.

3. For $h>14.8 \%$ there exist three fixed points of the dynamics. The two outer points are stable, and which of these is reached depends on the phenotypes initially present.

4. For $9.8 \%<h<14.8 \%$ and sufficiently high values of $r$ (Fig. 4), the attractor turns into a limit cycle, giving rise to Red Queen dynamics. On the other hand, for low values of $r$, the limit cycle breaks down and we recover the dynamical behaviour of cases 2 and 3 with the switch occurring at $h=12.6 \%$.

The boundary of region 4 is in fact slightly more complicated than the description above suggests because two further kinds of dynamics can occur here: (i) a limit-cycle attractor around each of the outer fixed points, and (ii) a limit-cycle attractor around all three fixed points with each of the outer fixed points also being an attractor. But the parameter space permitting these dynamics is very small compared to the others and they are therefore of less biological interest. We conclude that evolutionary cycling requires an intermediate harvesting efficiency plus prey evolution to occur sufficiently fast compared to predator evolution.

The results from the bifurcation analysis are intuitive in that evolutionary cycling requires: (i) the effect of selection by the predator on the prey to be great enough to drive the prey from the phenotypic equilibrium it would have in the absence of the predator ( $h$ not too low); (ii) sufficient need for the predator to track the prey's phenotypic change ( $h$ not too high); and (iii) in the resulting evolutionary race, the prey must be fast enough not to be "caught up" by the predator ( $r$ not too low). In view of the respiratory costs that the predators have to meet from consumption of prey simply to stay alive, one would expect $h$ to be substantially less than 1 and evolutionary cycling to occur in a range of $h$ likely to be observed in reality.

\section{MONOMORPHIC STOCHASTIC MODEL}

A realization of the monomorphic stochastic dynamics is given in Figs 3(c) and (d). The parameter values used are the same as those in Figs 3(a) and (b), and we see that the cyclic behaviour is still maintained. In addition, two major new effects should be noted. First, it can be seen that the oscillations in phenotypic values do not all have the same period. This phenomenon, which is well known in the theory of stochastic processes as phase diffusion (Tomita et al., 1974), comes about because stochastic perturbations along the limit cycle are not balanced by a counteracting force, whereas those orthogonal to the limit cycle are. Second, limit cycles whose extension in phenotype space is small relative to the typical mutational step sizes (given by $\sqrt{\operatorname{var} M_{1}}$ and $\sqrt{\operatorname{var} M_{2}}$ ) will be obscured by the stochastic noise. The boundaries of region 4 (Fig. 4) will then be less sharp than those in the monomorphic deterministic model. Thus, if the evolutionary cycling is to be visible, the mutational steps must not be too large. 


\section{POLYMORPHIC STOCHASTIC MODEL}

A realization of the polymorphic stochastic model is shown in Fig. 3(e) and (f), using as before the parameter values of Figs 3(a) and (b). The phenomenon of evolutionary cycling still persists despite the phenotypic distributions now being polymorphic. In addition, this model allows for the effects of demographic stochasticity also of the resident phenotypes (see Appendix). Although this superimposes more random variation to the solution, cycling is maintained. Provided that phenotypic variance is not too large and population sizes are not too small, we thus conclude that evolutionary cycling is robust to relaxation of the simplifying assumptions of the monomorphic deterministic model, and that it can actually occur in predator-prey communities such as the one defined in Section 2.

\section{Discussion}

The main result of this analysis is that evolutionary limit cycles, in which the predator and prey phenotypes continue to change indefinitely, are a natural outcome in a coevolutionary community. The cyclic behaviour is not an artefact of determinism or monomorphism, because the phenomenon can be observed both in the stochastic monomorphic simulations and in the stochastic polymorphic ones. Clearly, there is no general rule in nature to say that phenotypic evolution would lead to an equilibrium point in the absence of external changes in the environment.

A simple classification of the outcomes of phenotypic evolution can be constructed from two dichotomies. The first depends on whether an attractor exists, and the second on whether the attractor is a fixed point. This gives three classes of dynamics:

(i) evolution to a fixed-point attractor with stationary phenotypes,

(ii) evolution to an attractor that is not a fixed point on which the phenotypes continue to change indefinitely, and

(iii) evolution without an attractor, such that the phenotypes take more and more extreme values.

According to the definition in the Introduction, Red Queen dynamics would encompass classes (ii) and (iii). Class (iii) is unrealistic for most kinds of phenotypes and, if the Red Queen were to depend on the existence of such dynamics in nature, one could reasonably conclude that Red Queen dynamics would be very unusual (Rosenzweig et al., 1987). But this would be to miss class (ii), and dynamics of this kind we have shown here to be feasible. In fact, the limit cycle is but one of a number of non-equilibrium attractors; for instance in systems with more than two coevolving species, chaotic evolutionary attractors could be found.

Cyclic phenotype dynamics can occur in coevolution as is well known from theoretical studies of genetic polymorphisms under frequency-dependent selection (e.g. Akin, 1981; Seger, 1992), and research into the dynamics of strategy frequencies (e.g. Nowak \& Sigmund, 1989). The system considered here is different in two respects. First, the trait values are continuous, whereas cyclic dynamics have typically been observed in polymorphic systems with large qualitative differences between a small number of coexisting phenotypes. Second, and more important, the underlying genetic process here would be a sequence of gene substitutions in which mutants keep replacing the resident types rather than one in which the genes always coexist and undergo oscillations in frequency. Thus we are here looking at a process operating on an altogether larger evolutionary scale.

The monomorphic deterministic dynamic described here in fact turns out to be canonical (Dieckmann \& Law, 1995), and can be derived from other starting points such as quantitative genetics (Abrams et al., 1993). It seems, therefore, that there is a large class of models of phenotypic coevolution with the potential for non-equilibrium asymptotic states. This needs to be emphasized because the assumption that asymptotic states of evolution are fixed points underlies much contemporary evolutionary thought. This assumption and the techniques that go with it (in particular evolutionarily stable strategies) are clearly not appropriate for dealing with non-equilibrium asymptotic states. The prevailing view among evolutionary biologists, centred on equilibrium points, needs to be extended to a dynamical framework to assimilate the Red Queen.

This research was supported by the Royal Society (P.M.), the Evangelisches Studienwerk e. V. (U.D.), the Forschungszentrum Jülich GmbH F.R.G. (R.L. and U.D.) and a NERC equipment grant GR3/8205. We thank S. A. H. Geritz, V. A. A. Jansen, J. A. J. Metz and S. Mylius for discussions of the work.

\section{REFERENCES}

Abrams, P. A. (1986). Adaptive responses of predators to prey and prey to predators: the failure of the arms-race analogy. Evolution 40, 1229-1247.

Abrams, P. A., Matsuda, H. \& Harada, Y. (1993). Evolutionarily unstable fitness maxima and stable fitness minima of continuous traits. Evol. Ecol. 7, 465-487. 
AkIN, E. (1981). Cycling in simple genetic systems. J. math. Biol. 13, 305-324.

BROWN, J. S. \& VINCENT, T. L. (1992). Organization of predator-prey communities as an evolutionary game. Evolution 46, 1269-1283.

Cohen, J. E., Pimm, S. L., Yodzis, P. \& Saldan, J. (1993). Body sizes of animal predator and animal prey in food webs. J. Anim. Ecol. 62, 67-78.

Crawley, M. J. (ed.) (1992). Natural Enemies: The Population Biology of Predators, Parasites and Diseases. Oxford: Blackwell Scientific.

Dawkins, R. \& Krebs, J. R. (1979). Arms races between and within species. Proc. R. Soc. Lond. B 205, 489-511.

DieCKMANN, U. (1994) Coevolutionary dynamics of stochastic replicator systems. Diplomarbeit RWTH Aachen, Berichte des Forschungszentrums Jülich Jül-3018, Jülich Germany.

Dieckmann, U. \& Law, R. (1995). The dynamical theory of coevolution: a derivation from stochastic ecological processes. J. math. Biol., in press.

Fisher, R. A. (1958). The Genetical Theory of Natural Selection, 2nd edn. New York: Dover Publications.

GILlesPIE, D. T. (1976). A general method for numerically simulating the stochastic time evolution of coupled chemical reactions. J. comp. Phys. 22, 403-434.

Hofbauer, J. \& Sigmund, K. (1990). Adaptive dynamics and evolutionary stability. Appl. Math. Lett. 3, 75-79.

Marrow, P. (1992) The evolution and dynamics of interacting populations. D. Phil. thesis, University of York, York, U.K.

Marrow, P., Law, R. \& Cannings, C. (1992). The coevolution of predator-prey interactions: ESSs and Red Queen dynamics. Proc. Roy. Soc. Lond. B 250, 133-141.

Marrow, P. \& CANNINGS, C. (1993) Evolutionary instability in predator-prey systems. J. theor. Biol. 160, 135-150.

Marrow, P., Dieckmann, U. \& Law, R. (1995) Evolutionary dynamics of predator-prey systems: an ecological perspective. J. math Biol., in press.

Maynard Smith, J. \& Price, G. R. (1973). The logic of animal conflict. Nature, Lond. 246, 15-18.

Metz, J. A. J., Nisbet, R. M. \& Geritz, S. A. H. (1992). How should we define 'fitness' for general ecological scenarios? Trends Ecol. Evol. 7, 198-202.

NowaK, M. \& Sigmund, K. (1989). Oscillations in the evolution of reciprocity. J. theor. Biol. 137, 21-26.

PARKer, G. A. (1985). Population consequences of evolutionarily stable strategies. In: Behavioural Ecology: Ecological Consequences of Adaptive Behaviour (Sibly, R. M. \& Smith, R. H., eds) p. 35-58. Oxford: Blackwell Scientific.

RAND, D. A., Wilson, H. B. \& MCGlade, J. M. (1994) Dynamics and evolution: evolutionarily stable attractors, invasion exponents and phenotype dynamics. Phil. Trans. R. Soc. B 343, 261-283.

RosenZweig, M. L. (1973). Evolution of the predator isocline. Evolution 27, 84-94.

Rosenzweig, M. L., Brown, J. S. \& Vincent, T. L. (1987). Red Queens and ESS: the coevolution of evolutionary rates. Evol. Ecol. 1, 59-94.

Seger, J. (1992). Evolution of exploiter-victim relationships. In: Natural Enemies: The Population Biology of Predators, Parasites and Diseases (Crawley, M. J., ed) pp. 3-25. Oxford: Blackwell Scientific.

Serra, R., Andretta, M., Compiani, M. \& Zanarini, G. (1986). Introduction to the Physics of Complex Systems. Oxford: Pergamon Press.

Stenseth, N. C. \& Maynard Smith, J. (1984). Coevolution in ecosystems: Red Queen evolution or stasis? Evolution 38, 870-880.

TомiтA, T., Онта, T. \& Tоміта, H. (1974). Irreversible circulation and orbital revolution. Prog. theor. Phys. 52, 1744-1765.

van der LaAn, J. D. \& Hogeweg, P. (1995) Predator-prey coevolution: interactions across different timescales. Proc. R. Soc. Lond. B 259, 35-42.

van Kampen, N. G. (1981). Stochastic Processes in Physics and Chemistry. Amsterdam; North-Holland.

VAN VALEN, L. (1973). A new evolutionary law. Evol. Theory 1, 1-30.
VINCENT, T. L. (1991). Strategy dynamics and the ESS. In: Dynamics of Complex Interconnected Biological Systems (Vincent, T. L., Mees, A. I. \& Jennings, L. S., eds) pp. 236-262. Basel: Birkhauser.

Note added in proof: Since this paper was prepared, van der Laan \& Hogeweg (1995) have also considered a model of Red Queen dynamics incorporating population dynamic change.

\section{APPENDIX}

In this appendix we provide a brief derivation of the three dynamical models describing the process of coevolution for the reader interested in the more technical details. The theory outlined here is general in so far as it applies to a large variety of $N$-species coevolutionary communities of which the predatorprey system analyzed in this paper is just a particular instance.

\section{THE POLYMORPHIC STOCHASTIC MODEL}

At time $t$ there are $n_{i}$ individuals in species $i$ with $i=1, \ldots, N$. These have phenotypes $s_{i j}$ with $j=1, \ldots, n_{i}$ such that the phenotypic distribution $p_{i}\left(s_{i}\right)$ in species $i$ is given by

$$
p_{i}=\sum_{j=1}^{n_{i}} \delta_{s_{i j}}
$$

with $\delta_{x}(y)=\delta(x-y)$ where $\delta$ denotes Dirac's $\delta$-function. These distributions change in time due to stochastic birth, death and mutation processes like those specified in Table 1.

The dynamics of the phenotypic distributions can be described by a functional master equation for $P(p, t)$, the probability density of $p=\left(p_{1}, \ldots, p_{N}\right)$ to be realized at time $t$ (Dieckmann, 1994). The algorithm derived from this equation is the following.

1. Initialize the phenotypic distributions $p_{i}$ with $i=1, \ldots, N$ at time $t=0$ and specify the time $T$ when to stop the dynamics.

2. Calculate the birth and death probabilities $b_{i}\left(s_{i j}, p\right)$ and $d_{i}\left(s_{i j}, p\right)$ for each individual $i=1, \ldots, N$, $j=1, \ldots, n_{i}$ with phenotype $s_{i j}$ in the environment given by $p$.

Remark. According to Table 1 we have for the predator-prey community

$$
\begin{gathered}
b_{1}\left(s_{1}, p\right)=r_{1}, \\
d_{1}\left(s_{1}, p\right)=\int \alpha\left(s_{1}\right) p_{1}\left(\tilde{s}_{1}\right) d \tilde{s}_{1}+\int \beta\left(s_{1}, s_{2}\right) p_{2}\left(s_{2}\right) d s_{2}, \\
b_{2}\left(s_{2}, p\right)=\int \gamma\left(s_{1}, s_{2}\right) p_{1}\left(s_{1}\right) d s_{1}, \text { and } d_{2}\left(s_{2}, p\right)=r_{2} .
\end{gathered}
$$


3. Construct the sums

$$
\begin{gathered}
w_{i j}=b_{i}\left(s_{i j}, p\right)+d_{i}\left(s_{i j}, p\right), \\
w_{i}=\sum_{j=1}^{n_{i}} w_{i j}
\end{gathered}
$$

and

$$
w=\sum_{i=1}^{N} w_{i}
$$

with $i=1, \ldots, N, j=1, \ldots, n_{i}$.

4. Choose the waiting time $\Delta t$ for the next event to occur according to

$$
\Delta t=-\frac{1}{w} \ln r
$$

where $0<r \leqslant 1$ is a uniformly distributed random number.

5. Choose species $i$ with probability

$$
\frac{1}{w} w_{i}
$$

Choose individual $j$ in species $i$ with probability

$$
\frac{1}{w_{i}} w_{i j} \text {. }
$$

Choose then a birth or death event with probability

$$
\frac{1}{w_{i j}} b_{i}\left(s_{i j}, p\right) \text { and } \frac{1}{w_{i j}} d_{i}\left(s_{i j}, p\right)
$$

respectively.

6. If a birth event occurs for an individual with phenotype $s_{i j}$, choose a new phenotype $s_{i j}^{\prime}$ with probability density

$$
\left(1-\mu_{i}\right) \delta\left(s_{i j}^{\prime}-s_{i j}\right)+\mu_{i} M_{i}\left(s_{i j}^{\prime}-s_{i j}\right) .
$$

7. Update time and phenotypic distributions according to $t \rightarrow t+\Delta t$ and $p_{i} \rightarrow p_{i}+\delta_{s_{i j}^{\prime}}$ or $p_{i} \rightarrow p_{i}-\delta_{s_{i j}}$ for a birth or death event in species $i$ respectively.

8. Continue from Step 2 until $t \geqslant T$.

The protocol above utilizes the minimal process method (Gillespie, 1976) to simulate the functional master equation. Note in particular that according to Step 4 the waiting times follow an exponential distribution, the standard result for stochastic processes described by homogeneous master equations.
THE MONOMORPHIC STOCHASTIC MODEL

If $\mu_{i}$ is sufficiently small for all $i=1, \ldots, N$, the phenotypic distributions in each species will be sharply concentrated around a single phenotype, the resident phenotype. The distributions then are called monomorphic (precisely, one should refer to them as quasi-monomorphic since still more than one phenotype may be present in the population) and can well be approximated by $p_{i}=n_{i} \delta_{s_{i}}$ with resident phenotype $s_{i}$ and population size $n_{i}$. In this case phenotypic change occurs via a sequence of trait substitutions where a resident phenotype $s_{i}$ is replaced by a mutant phenotype $s_{i}^{\prime}$ (Dieckmann, 1994).

The resulting directed random walk in the phenotype space is described by the master equation

$$
\begin{aligned}
& \frac{\mathrm{d}}{\mathrm{d} t} P(s, t) \\
& \quad=\int\left[w\left(s \mid s^{\prime}\right) P\left(s^{\prime}, t\right)-w\left(s^{\prime} \mid s\right) P(s, t)\right] \mathrm{d} s^{\prime},
\end{aligned}
$$

where $P(s, t)$ denotes the probability density of the resident phenotypes to be given by $s=\left(s_{1}, \ldots, s_{N}\right)$ at time $t$. Equation (A.2) only holds if the stochastic dynamics of $s$ are a Markov process; to guarantee this it can be necessary to consider more than one trait per species. This more general case is analysed in Dieckmann \& Law (1995). In the infinitesimal time interval $\mathrm{d} t$, a trait substitution in only a single trait can occur and thus the probability per unit time $w\left(s^{\prime} \mid s\right)$ for the transition $s \rightarrow s^{\prime}$ can be decomposed according to

$$
w\left(s^{\prime} \mid s\right)=\sum_{i=1}^{n} w_{i}\left(s_{i}^{\prime}, s\right) \prod_{\substack{j=1 \\ j \neq i}}^{n} \delta\left(s_{j}^{\prime}-s_{j}\right) .
$$

Here $w_{i}\left(s_{i}^{\prime}, s\right)$ denotes the probability per unit time for a trait substitution $s_{i} \rightarrow s_{i}^{\prime}$ to occur in species $i$ given an environment of phenotypes $s$.

A trait substitution requires that, first, a specific mutant phenotype $s_{i}^{\prime}$ enters the population of species $i$ and, second, that it succeeds in replacing the resident phenotype $s_{i}$. Since these two processes are statistically independent, their probabilities multiply and $w_{i}\left(s_{i}^{\prime}, s\right)$ is given by the product

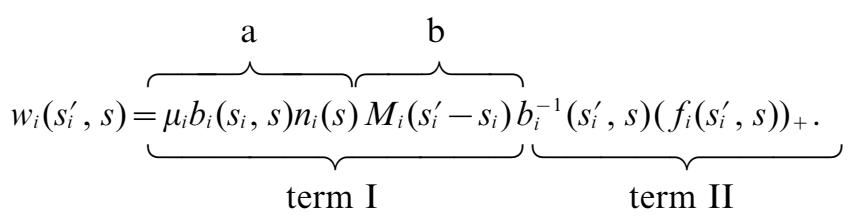


The functions $b_{i}\left(s_{i}^{\prime}, s\right), \quad d_{i}\left(s_{i}^{\prime}, s\right)$ and $f_{i}\left(s_{i}^{\prime}, s\right)=$ $b_{i}\left(s_{i}^{\prime}, s\right)-d_{i}\left(s_{i}^{\prime}, s\right)$ denote the per capita birth, death and growth probabilities per unit time (or rates) of a phenotype $s_{i}^{\prime}$ in an environment given by the phenotypes $s$. They are defined in terms of the analogous quantities of the polymorphic stochastic model by e.g. $b_{i}\left(s_{i}^{\prime}, s\right)=b_{i}\left(s_{i}^{\prime}, p\right)$, with $p=\left(n_{1}(s) \delta_{s_{1}}, \ldots, n_{N}(s) \delta_{s_{N}}\right)$. Here the population sizes $n_{i}(s)$ are determined as the equilibrium solutions of the resident's population dynamics

$$
\frac{\mathrm{d}}{\mathrm{d} t} n_{i}=n_{i} f_{i}\left(s_{i}, s\right) .
$$

The general case of nonequilibrium population dynamics is treated in Dieckmann \& Law (1995). A more formal analysis is given in Rand et al. (1993).

Remark. For the predator-prey community, we obtain $\quad b_{1}\left(s_{1}^{\prime}, s\right)=r_{1}, \quad d_{1}\left(s_{1}^{\prime}, s\right)=\alpha\left(s_{1}^{\prime}\right) n_{1}(s)+$ $\beta\left(s_{1}^{\prime}, s_{2}\right) n_{2}(s), b_{2}\left(s_{2}^{\prime}, s\right)=\gamma\left(s_{1}, s_{2}^{\prime}\right) n_{1}(s)$, and $d_{2}\left(s_{2}^{\prime}, s\right)=$ $r_{2}$.

We now explain the different terms in equation (A.4).

1. Term I represents the impact of the mutation process and is given by weighting (a) the probability per unit time for any mutant to occur in the resident population of phenotype $s_{i}$ by (b) the probability density for the mutant phenotype to be given by $s_{i}^{\prime}$. Since mutations in distinct individuals are statistically uncorrelated, term Ia is composed of three factors. The per capita birth rate $b_{i}\left(s_{i}, s\right)$ of the resident phenotype is multiplied by $\mu_{i}$, the mutation probability for each birth event, giving the per capita mutation rate of the resident phenotype. This is multiplied by the resident's population size $n_{i}$ to yield the mutation rate of the resident population. Term $\mathrm{Ib}$ simply is the mutation distribution $M_{i}\left(s_{i}^{\prime}-s_{i}\right)$ for mutant phenotypes $s_{i}^{\prime}$ given the resident phenotype $s_{i}$.

2. Term II stands for the process of selection. In the monomorphic stochastic model it is assumed that the resident populations are sufficiently large not to be subject to accidental extinction due to stochastic fluctuations of their population sizes. In contrast, the mutant population starts with population size 1 such that the impact of demographic stochasticity on its dynamics ought to be considered. Since the function ()$_{+}$returns its argument if the argument is positive and 0 otherwise, deleterious mutants, with $f_{i}\left(s_{i}^{\prime}, s\right)<0$, have no chance to survive in the resident population. But even advantageous mutants, with $f_{i}\left(s_{i}^{\prime}, s\right)>0$, experience some risk of accidental extinction due to random sampling when initially rare (Fisher, 1958). Term II also shows that for large initial per capita growth rates of the mutant, the probability for it to succeed in replacing the resident saturates at 1 . The exact form of term II is derived in Dieckmann \& Law (1995).

Combining eqns (A.2), (A.3) and (A.4) yields a complete description of the stochastic coevolutionary dynamics provided that phenotypic distributions are sufficiently concentrated and that population sizes are sufficiently large. The algorithm for this model again follows the minimal process method.

\section{THE MONOMORPHIC DETERMINISTIC MODEL}

To capture the representative features of the monomorphic coevolutionary dynamics directly, rather than having to consider a large number of different realizations of the monomorphic stochastic model, a deterministic approximation of the latter is devised. The deterministic path $\langle s\rangle$ associated with a stochastic process is described by the equation (Serra et al., 1986).

$$
\frac{\mathrm{d}}{\mathrm{d} t}\langle s\rangle=a(\langle s\rangle),
$$

where the function $a$ is the first jump moment of the stochastic process whose components in our case are given by

$$
a_{i}(s)=\int\left(s_{i}^{\prime}-s_{i}\right) w_{i}\left(s_{i}^{\prime}, s\right) \mathrm{d} s_{i}^{\prime},
$$

with $i=1, \ldots, N$. If the different realizations of the stochastic process do not spread too far apart, i.e. if the variance of the probability density $P(s, t)$ in eqn (A.2) stays small, the deterministic path $\langle s\rangle$ provides a good approximation of the mean path

$$
\int s P(s, t) \mathrm{d} s
$$

(van Kampen, 1981).

We obtain the deterministic monomorphic model by introducing eqn (A.4) into (A.6). To simplify the result, we expand the functions $f_{i}\left(s_{i}^{\prime}, s\right)$ and $b_{i}^{-1}\left(s_{i}^{\prime}, s\right)$ in the mutant phenotype $s_{i}^{\prime}$ about the resident phenotype $s_{i}$. Here we only present the first order result for symmetric mutation distributions, the derivation of higher order correction terms and for arbitrary mutation distributions is given in Dieckmann \& Law (1995). By introducing the result into eqn (A.5), we obtain the deterministic path of the monomorphic stochastic model 


$$
\frac{\mathrm{d}}{\mathrm{d} t}\left\langle s_{i}\right\rangle \underbrace{\left(\frac{1}{2} \mu_{i} \operatorname{var} M_{i}\right) n_{i}(\langle s\rangle)}_{\text {term I }} \underbrace{\left.\frac{\partial}{\partial s_{i}^{\prime}} f_{i}\left(s_{i}^{\prime},\langle s\rangle\right)\right|_{s_{i}^{\prime}=\left\langle s_{i}\right\rangle},}_{\text {term II }}
$$

for $i=1, \ldots, N$. The deterministic path thus is described by a simple, though typically nonlinear, dynamical system composed of $N$ coupled first order differential equations. The terms on the right-hand side of eqn (A.7) have the following meanings.

1. Term I again captures the influence of mutation on the coevolutionary dynamics. The factor $\frac{1}{2} \mu_{i} \operatorname{var} M_{i}$, called the evolutionary rate constant, is affected by the proportion $\mu_{i}$ of births that produce mutants and by the variance $\operatorname{var} M_{i}$ of the mutation distribution in the trait $s_{i}$. Together with the population size $n_{i}$, all these terms are non-negative, so term I as a whole serves to scale the rate of evolutionary change.

2. Term II accounts for the impact of selection as it determines the direction of evolutionary change. When this derivative of the per capita growth rate $f_{i}$ is positive (respectively negative), mutants with increased (respectively decreased) phenotypic values $s_{i}$ will be advantageous in the environment given by $\langle s\rangle$. The lines on which the terms II are zero are the isoclines of the monomorphic deterministic dynamics.

The Runge-Kutta method can be employed to construct an algorithm for the monomorphic deterministic model. Equations (A.7) have features in common with other models of adaptive dynamics (Hofbauer \& Sigmund, 1990; Vincent, 1991; Abrams et al., 1993), but are here explicitly derived from the underlying stochastic ecological processes. 